\title{
Compact GNSS Metasurface-inspired Cavity Antennas
}

\author{
Laura Garcia-Gamez, Loïc Bernard, Member, IEEE, Sylvain Collardey, Member, IEEE, Hrvoje Covic, \\ Ronan Sauleau, Fellow, IEEE, Kourosh Mahdjoubi, Patrick Potier and Philippe Pouliguen
}

\begin{abstract}
This paper presents an innovative circularlypolarized compact cavity antenna based on metasurfaces; the proposed design is explained starting from a linearly-polarized antenna based on similar concepts. The main objective is to cover three different GNSS systems, namely Galileo E1, GLONASS G1 and GPS L1, with a single antenna embedded in a metallic cavity. The aperture dimension is set to $0.26 \lambda_{0} \times 0.26 \lambda_{0}$, with a central frequency of $1578 \mathrm{MHz}$. Loading the aperture with a metasurface allows an efficient radiation within such a small aperture size. Our experimental results are in very good agreement with the simulations, with an axial ratio lower than $2 \mathrm{~dB}$ between $1540 \mathrm{MHz}$ and $1655 \mathrm{MHz}$.
\end{abstract}

Index Terms - circular polarization, compact antenna, GNSS, metasurface.

\section{INTRODUCTION}

$\mathrm{M}$ INIATURE antennas have been studied for a plethora of applications, like mobile communication, radionavigation or geolocation systems. They are also used for accurate positioning and tracking of fastly-flying vehicles (e.g. projectiles). In many configurations, such antennas are embedded in compact metallic cavities with a typical aperture size smaller than $0.3 \times \lambda_{0}$, where $\lambda_{0}$ is the wavelength in vacuum at the operating frequency; this strategy allows to guarantee its mechanical robustness and maintain the carrier aerodynamic properties. In practice, the radionavigation antenna module (which operates in right-hand circular polarization (RHCP)) must cooperate with at least three GNSS systems, namely the L1 GPS, E1 Galileo and G1 Glonass bands, whose carrier frequencies equal 1575.42 MHz for GPS and Galileo, and 1602 $\mathrm{MHz}$ for Glonass. This corresponds to a minimum acceptable antenna bandwidth of $60 \mathrm{MHz}(3.7 \%$ at $1600 \mathrm{MHz})$.

Various compact antennas have been proposed for GNSS applications, e.g. [2]-[5]. Compact circularly-polarized (CP) configurations have been designed in [4] and [5] at $1.6 \mathrm{GHz}$, their dimensions equal $0.373 \lambda_{0} \times 0.373 \lambda_{0} \times 0.016 \lambda_{0}$ and $0.32 \lambda_{0} \times 0.32 \lambda_{0} \times 0.026 \lambda_{0}$, respectively. An even more compact structure $\left(0.177 \lambda_{0} \times 0.1810 \lambda_{0} \times 0.025 \lambda_{0}\right)$ has been proposed in [6], with a radiation efficiency around $72 \%$. Nevertheless, none of these solutions are integrated into metallic cavities. Such a constrained environment has a very strong impact on the overall antenna performance [7]; in particular, it is responsible for a significant reduction of the antenna bandwidth. Various

This work was funded in part by ISL (French-German Research Institute of Saint-Louis) and DGA (Direction Générale de l'Armement).

L. Garcia-Gamez, L. Bernard and H. Covic are with the French-German Research Institute of Saint-Louis, 68301 Saint Louis, France (e-mail: laura.garcia-gamez@isl.eu). attempts have been proposed to overcome this limitation. In [8], a stacked linearly-polarized (LP) configuration is introduced to enhance the antenna bandwidth by a factor 3.3, compared to a single-layer patch. Similarly the stacked-patch configuration proposed in [9] exhibits a $-10-\mathrm{dB}$ reflection bandwidth of $23 \%$. The LP magneto-electric dipole antenna integrated into a lowprofile cavity described in [10] exhibits an impedance bandwidth of 54\%. Artificial magnetic conductors (AMC) have been investigated in [11] to design a low profile $\mathrm{CP}$ antenna, with a thickness lower than $0.1 \times \lambda_{0}$; an axial ratio bandwidth of $33.2 \%$ has been achieved with an impedance bandwidth of $36.2 \%$. Finally, the quality of circular polarization can be improved [12] by employing a parasitic patch with an aperture antenna, leading to an impedance bandwidth of more than $70 \%$ and a $3-\mathrm{dB}$ axial ratio bandwidth of $43.3 \%$. Nevertheless, in all reported studies, the minimum antenna aperture size is in the order of $0.6 \lambda_{0} \times 0.6 \lambda_{0}$, or larger (the overall antenna dimensions equal $0.967 \lambda_{0} \times 0.967 \lambda_{0} \times 0.173 \lambda_{0}, 0.72 \lambda_{0} \times 0.60 \lambda_{0} \times 0.19 \lambda_{0}$, and $0.80 \lambda_{0} \times 0.80 \lambda_{0} \times 0.30 \lambda_{0}$ in [10], [11] and [12] respectively).

Bandwidth limitations of small cavity antennas have been studied in [13]-[16]. In particular, it has been demonstrated in [15] and [16] that stacked patch antennas cannot exhibit the desired bandwidth (at least $60 \mathrm{MHz}$ here) when embedded in a metallic cavity of size $0.245 \lambda_{0} \times 0.245 \lambda_{0} \times 0.115 \lambda_{0}$. Metasurfaceinspired solutions have thus been proposed by the authors to approach the theoretical bounds defined in [17], and a LP metasurface antenna has been first introduced in [13] as a practical implementation (at the frequency of $1575 \mathrm{MHz}$ ) of the theoretical developments given in [17].

We propose here two compact metasurface-inspired antennas operating at $1578 \mathrm{MHz}$ and embedded in a very small square metallic cavity of size $0.26 \lambda_{0}$. The first one, linearly-polarized, is briefly described in Section II to better explain the operation principle of the RHCP counterpart that is excited by four separate feed ports (Section III). Conclusions are drawn in Section IV.

\section{LINEARLY-POLARIZED ANTENNA}

\section{A. Antenna geometry}

The antenna geometry, provided in Fig. 1, is embedded in a square cavity whose dimensions $\left(50 \times 50 \times 20 \mathrm{~mm}^{3}\right.$, i.e. $\left.0.26 \lambda_{0} \times 0.26 \lambda_{0} \times 0.11 \lambda_{0}\right)$ are dictated by the flying platform

R. Sauleau, K. Mahdjoubi and S. Collardey are with Univ Rennes, CNRS, IETR (Institut d'Électronique et de Télécommunications de Rennes) - UMR 6164, F-35000 Rennes, France (e-mail: ronan.sauleau@univ-rennes1.fr).

P. Potier and P. Pouliguen are with the Direction Générale de l'Armement Maîtrise de l'Information, 35170 Bruz, France. 
diameter (around $150 \mathrm{~mm}$ ). This design is inspired from [16], but with a larger cavity size.

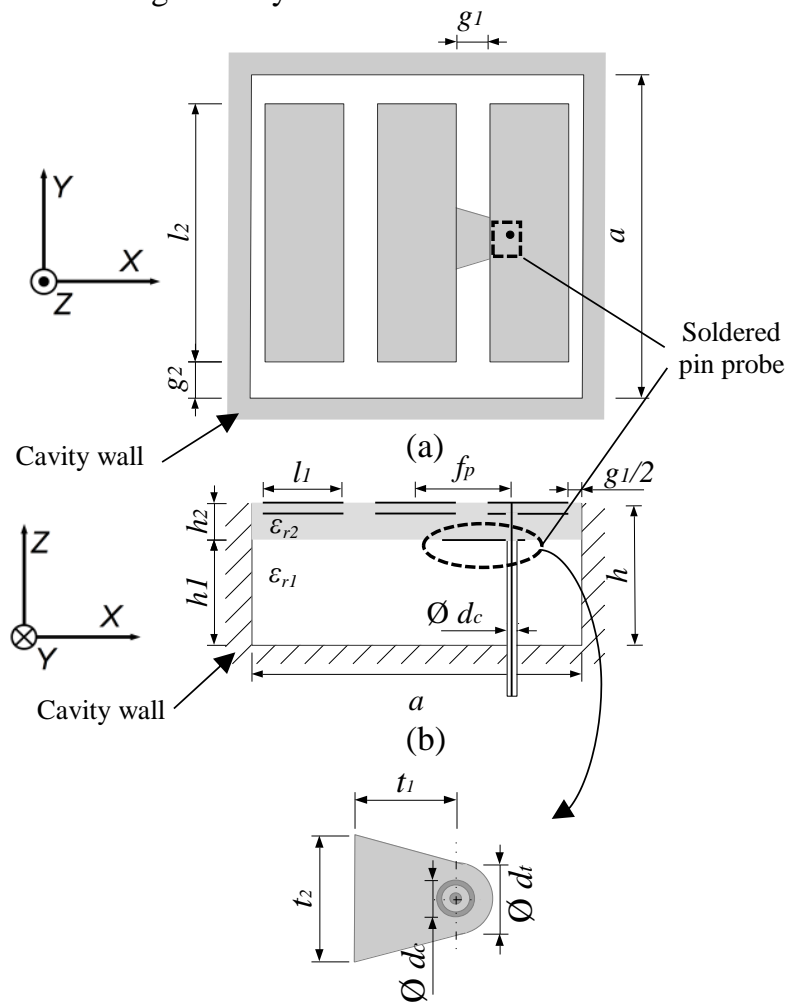

(c)

Fig. 1. LP antenna embedded in a square cavity. (a) Top view. (b) Cross-section view. (c) Feeding system.

The metallic cavity is filled with two different materials, as shown in Fig. 1b:

- A thick polypropylene layer $\left(\varepsilon_{r 1}=2.25, \tan \delta_{1}=0.001, h_{1}=\right.$ $18.08 \mathrm{~mm}$, [18]) selected to guaranty an adequate robustness to the structure, especially due to the strong acceleration constraints supported by the flying platforms (several thousands of $g$ ),

- A thin lamination of two Rogers RO3210 substrates $\left(\varepsilon_{r 2}=\right.$ 10.2, $\tan \delta_{2}=0.0027, h_{2}=1.92 \mathrm{~mm}$ ) with three metallic levels: the lowest one is the feeding layer with a trapezoidal shape transmission line (Fig. 1c), while the metasurface-inspired radiating element is printed on the two upper metallic layers; the upper substrate is thin enough $(0,64 \mathrm{~mm})$ to provide the requested capacitive effect [17] and thus enlarge the antenna bandwidth; both upper metallic layers have identical patterns made of three rectangular strips of equal size $\left(l_{1} \times l_{2}\right)$. These dimensions are smaller than $\lambda_{0} / 10$, which satisfies the metasurface periodicity and size criteria. Moreover, the capacitance loading of the radiating aperture can be adjusted by tuning the gap values $g_{1}$ and $g_{2}$ (Figs. $1 \mathrm{a}$ and $1 \mathrm{~b}$ ).

\section{B. Numerical and experimental results}

All simulations have been carried out using CST Studio Suite [19]. The glue parameters (estimated to $\varepsilon_{r}=3.5, \tan \delta=0.01, h$ $=0.1 \mathrm{~mm}$ ) have been taken into account in the simulation results. As design guidelines, first, the strip width $\left(l_{1}\right)$ is chosen to be about $\lambda_{0} / 13$ (which also determines $g_{1}$ ), and $l_{2}$ is fixed to
$0.77 \times a$ to get a wide enough bandwidth [17]. The antenna center frequency and matching level are then tuned by the variables $f_{p}$ and finely optimized by adjusting the dimensions $t_{1}$ and $t_{2}$ (Fig. 1c) [16].

TABLE I. OPTIMIZED DIMENSIONS OF THE LP ANTENNA

\begin{tabular}{cccc}
\hline \hline Parameter & Value $(\mathrm{mm})$ & Parameter & Value $(\mathrm{mm})$ \\
\hline$a$ & 50 & $l_{2}$ & 38.8 \\
$h_{l}$ & 18.08 & $\varepsilon_{\mathrm{r} 1}$ & 2.25 \\
$h_{2}$ & 1.92 & $\varepsilon_{\mathrm{r} 2}$ & 10.2 \\
$f_{p}$ & 13.2 & $t_{1}$ & 8.8 \\
$g_{I}$ & 3.83 & $t_{2}$ & 8.2 \\
$g_{2}$ & 5.6 & $d_{t}$ & 4.22 \\
$l_{l}$ & 12.77 & $d_{\mathrm{c}}$ & 2.2 \\
\hline \hline
\end{tabular}

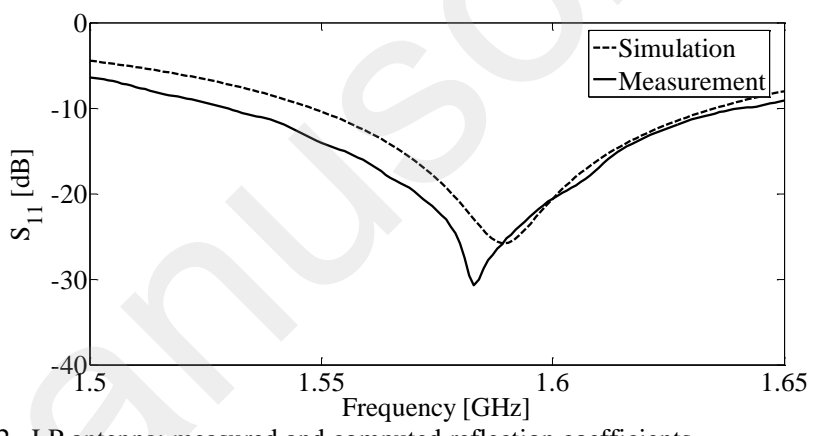

Fig. 2. LP antenna: measured and computed reflection coefficients.

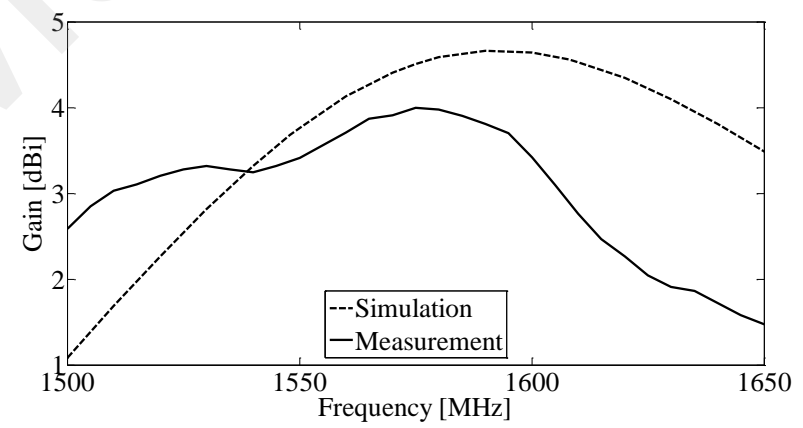

Fig. 3. LP antenna: measured and computed gains (at broadside).

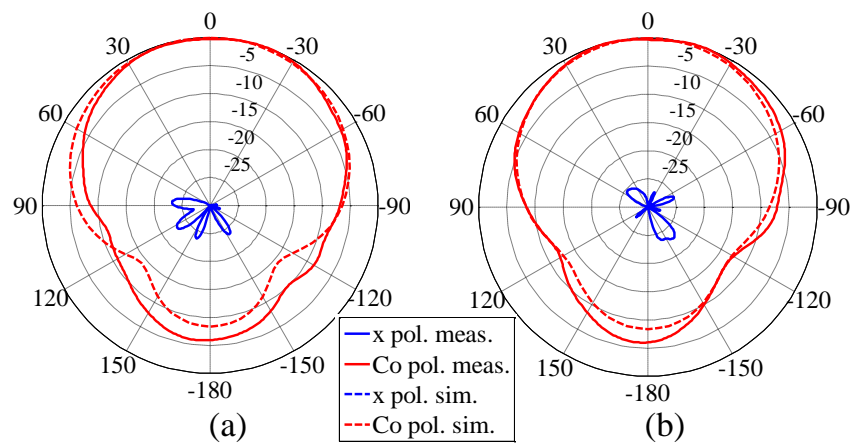

Fig. 4. LP antenna: measured (solid line) and computed (dotted line) normalized radiation patterns in $\mathrm{dBi}$ at $1578 \mathrm{MHz}$ in two vertical cut planes: (a) $\varphi=0^{\circ}$ (E-plane), (b) $\varphi=90^{\circ}$ (H-plane).

The final dimensions are provided in Table I. The simulated and measured reflection coefficients, gains and radiation patterns are in good agreement with each other as confirmed by Fig. 2, Fig. 3 and Fig. 4 respectively. The antenna is well matched over the three aimed GNSS bands. The peak gain 
reaches $4 \mathrm{dBi}$ and the half power beam width (HPBW) is about $130^{\circ}$ in all vertical cut planes.

\section{CIRCULARLY-POLARIZED ANTENNA}

\section{A. Antenna geometry}
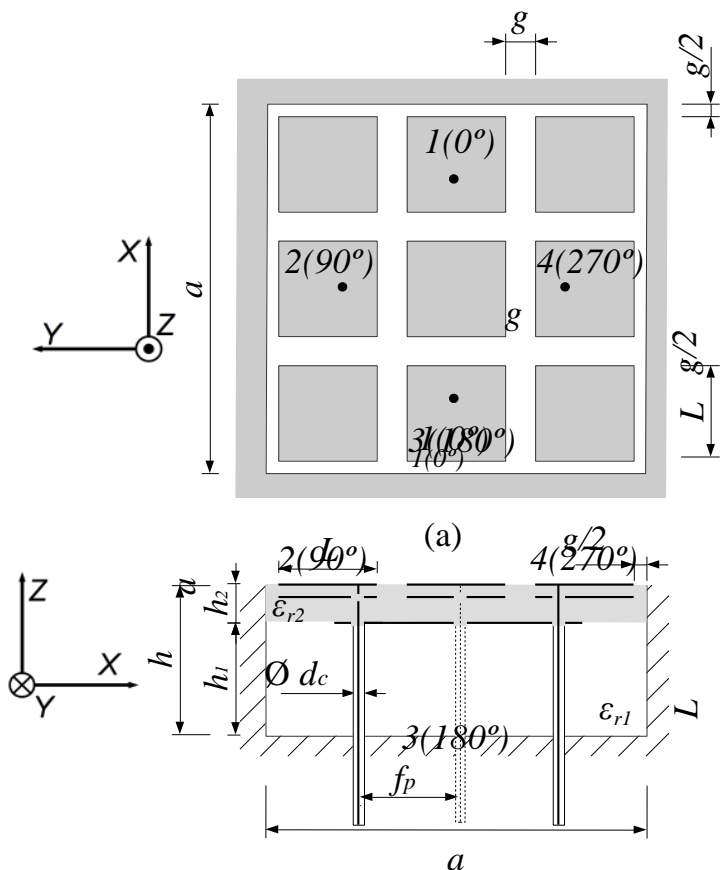

(b)

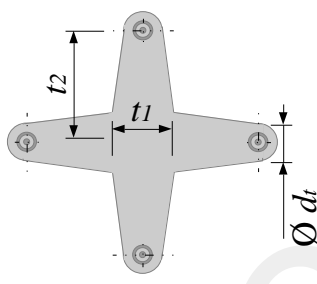

(c)

Fig. 5. CP antenna embedded in a square cavity. (a) Top view. (b) Cross-section view. (c) Feeding system.

The LP antenna design is extended to circular polarization (CP) by replacing the two-layer rectangular strips loading the radiating aperture (Fig. 1a) by two arrays of $3 \times 3$ capacitivelycoupled square patches $(L \times L)$, as illustrated in Fig. 5a. The dielectric stack-up (Fig. 5b) is similar to the one used in LP (Fig. 1b). The antenna module is fed by four independent ports (as shown in Fig. 5) excited in phase quadrature [20], [21]. The outer conductors of each coaxial probe are soldered to a starshape feed (Fig. 5c).

\section{B. Design guidelines}

The gap $g$ between the square patches is a fundamental design parameter since it allows tuning the metasurface capacitance loading the radiating aperture, thus the resonant frequency of the antenna and its efficiency. The feed parameters $\left(f_{p}, t_{1}, t_{2}\right.$, Figs. $5 \mathrm{~b}$ and $\left.5 \mathrm{c}\right)$ are also optimized to improve the antenna matching level and impedance bandwidth. The final dimensions of the optimized prototype are provided in Table II. Figs. 6 and 7 illustrate the influence of two critical design parameters ( $g$ and $f_{p}$ respectively) on the reflection coefficient at one of the four input ports, assuming that all other dimensions are fixed and equal to their optimal values specified in Table II. Note that, by symmetry, the reflection coefficients are the same on each input port. Fig. 6 confirms the very strong impact of $g$ on the resonance frequency, while Fig. 7 shows that $f_{p}$ has a less important effect on the resonance frequency values, but a stronger impact of the matching level.

TABLE II. FOUR-PORTS CP ANTENNA OPTIMIZED PARAMETERS

\begin{tabular}{cccc}
\hline \hline Parameter & Value $(\mathrm{mm})$ & Parameter & Value $(\mathrm{mm})$ \\
\hline$a$ & 50 & $\varepsilon_{\mathrm{r} 1}$ & 2.25 \\
$h_{l}$ & 18.08 & $\varepsilon_{\mathrm{r} 2}$ & 10.2 \\
$h_{2}$ & 1.92 & $t_{l}$ & 8 \\
$f_{p}$ & 13.5 & $t_{2}$ & 13.5 \\
$g$ & 5 & $d_{t}$ & 4 \\
$L$ & 11.6 & $d_{\mathrm{c}}$ & 2.2 \\
\hline \hline
\end{tabular}

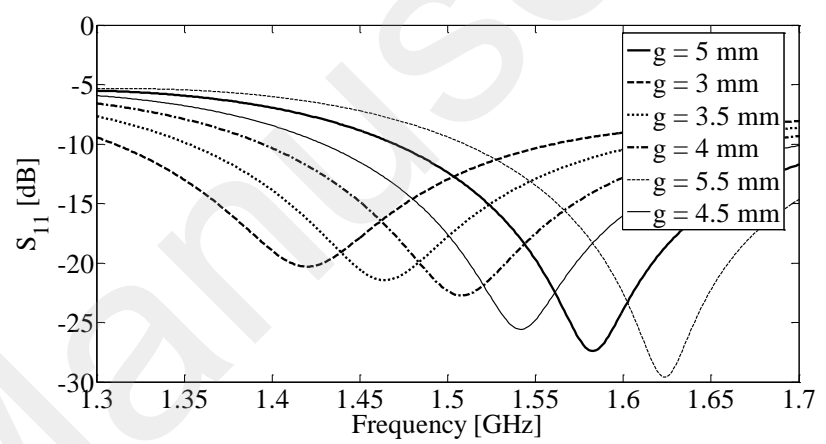

Fig. 6. Influence of parameter $g$ on the reflection coefficient of the antenna (all other parameter values are given in Table II).

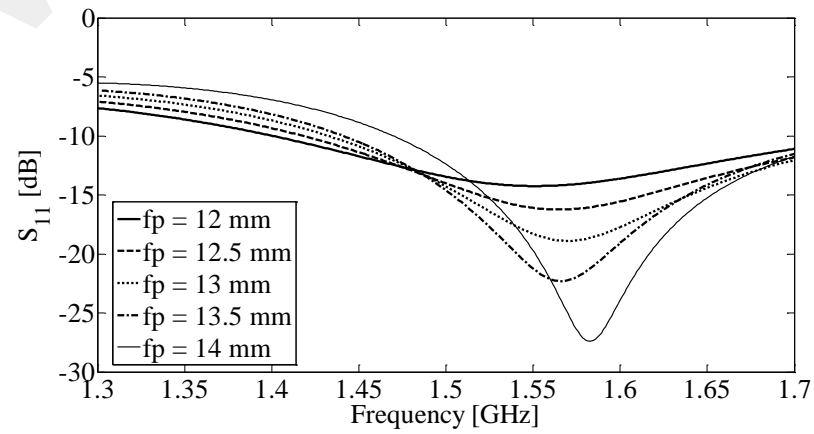

Fig. 7. Influence of parameter $f_{p}$ on the reflection coefficient of the antenna (all other parameter values are given in Table II).

\section{Prototyping and experimental results}

The antenna prototype (Figs. 8a and $8 \mathrm{~b}$ ) has been manufactured and measured. As for the LP antenna, thin layers of liquid dielectric glue have been used to assemble both dielectric layers and attach the antenna stack-up to the cavity walls. The four coaxial probes used to excite the antenna module are soldered as defined in Figs. 5a and 5b.

The measured and computed scattering parameters are represented in Fig. 9. $S_{11}, S_{22}, S_{33}$ and $S_{44}$ represent the measured reflection coefficients at each antenna port (Fig. 5a), while $S_{\text {ii }}$ represents the simulated result. $S_{\text {aa }}$ corresponds to the measured reflection coefficient at the output of the active combiner circuit connected to the antenna ports (see below). The antenna is well matched at each port, and the slight frequency shift observed between simulations and measurements is attributed to mechanical tolerances and material characteristics (e.g. losses 
in glue among others). The measured $-10-\mathrm{dB}$ reflection bandwidth is about $260 \mathrm{MHz}$. This quite large bandwidth (compared to the LP antenna) is attributed to the strong mutual coupling between ports, especially between two opposite ports, as shown in [22].

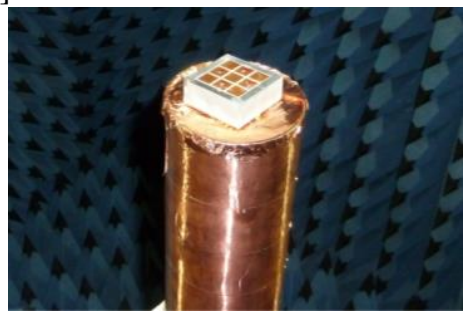

(a)

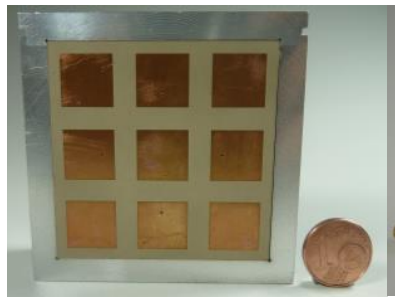

(b)

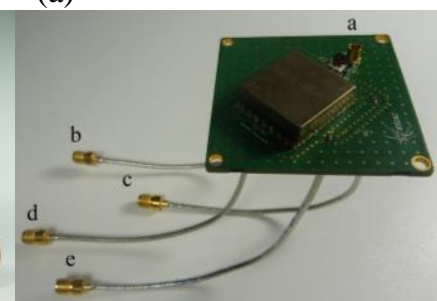

(c)
Fig. 8. Antenna prototype. (a) Antenna mounted on the rotating mast of the near-field anechoic chamber. (b) Top view. (c) Combiner system with four independent ports.

The feeding circuit, used to assess the antenna performance in CP, is shown in Fig. 8c. It is based on a commercial surface mounted four-port power splitter/combiner SCQ-4-1650+ [23] cascaded with a low noise amplifier BGA725L6 [24] developed for GNSS applications. The LNA is biased using the bias tee TCBT-6G+ [25]. This feeding system size has a total footprint of $60 \times 60 \mathrm{~mm}^{2}$. It has been characterized in amplitude and phase. The results, not shown here for the sake of brevity, show a maximum phase difference of $\pm 3^{\circ}$ between all ports from 1300 to $1700 \mathrm{MHz}$, while maintaining a very low amplitude imbalance.

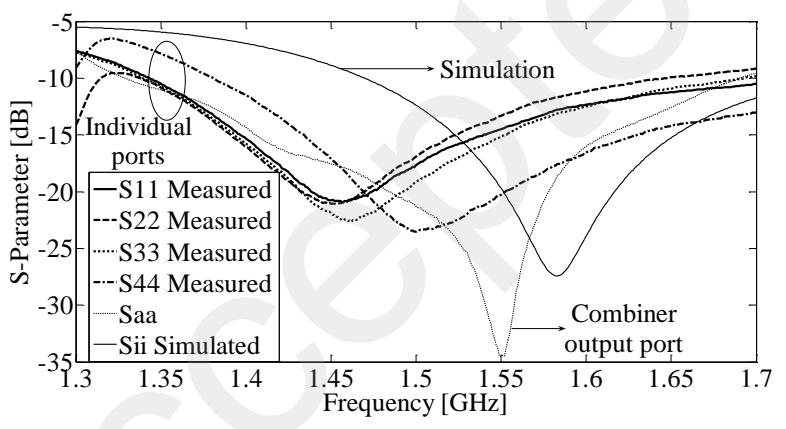

Fig. 9. Reflection coefficients measured at each input port of the $\mathrm{CP}$ antenna, and comparison with the simulated data. The reflection coefficient measured at the combiner out port is also shown.

The total gain of the antenna module, including the LNA gain, is plotted in Fig. 10 (left scale). Its average value is around $15 \mathrm{dBic}$ at the center frequency. The axial ratio measured at broadside (Fig. 10, right scale) remains below $3 \mathrm{~dB}$ between $1380 \mathrm{MHz}$ and more than $1700 \mathrm{MHz}$, and below $2 \mathrm{~dB}$ between $1540 \mathrm{MHz}$ and $1655 \mathrm{MHz}$. The axial ratio value at $45^{\circ}$ in elevation remains below $3 \mathrm{~dB}$ between $1500 \mathrm{MHz}$ and 1650 $\mathrm{MHz}$.
The normalized radiation patterns measured and simulated at $1578 \mathrm{MHz}$ are plotted in Fig. 11 in RHCP (co-polarization) and LHCP (cross-polarization). The agreement between simulations and experiments is very good, with a very low cross-polarization discrimination level $(<18 \mathrm{~dB}$ for elevation angles smaller than $60^{\circ}$ ).

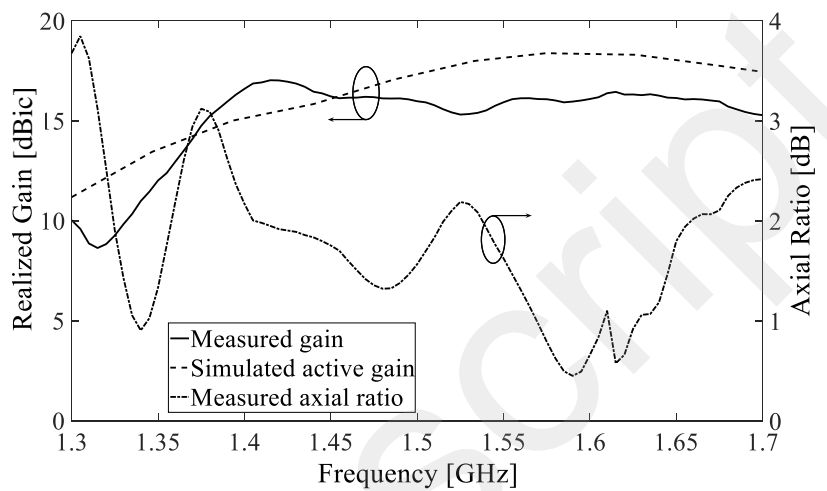

Fig. 10. Performance of the antenna module with its feeding system (Fig. 8c). Left: measured and computed gain. Right: measured axial ratio.

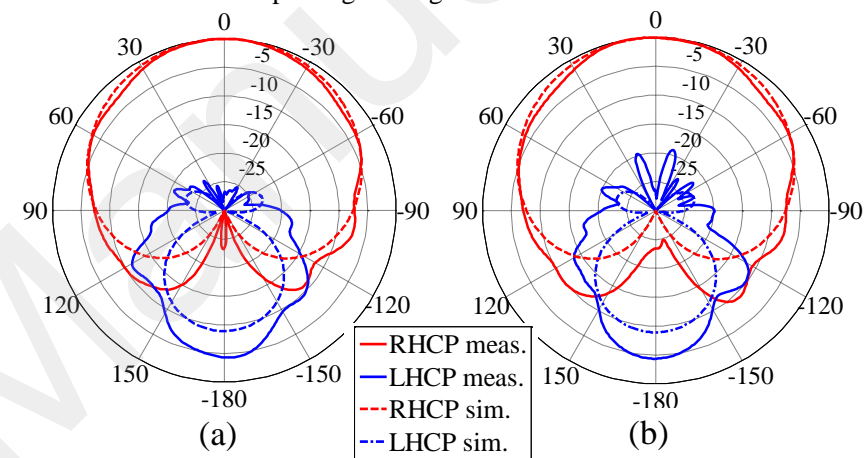

Fig. 11. CP antenna: measured (solid line) and computed (dotted line) normalized radiation patterns in $\mathrm{dBic}$ at $1578 \mathrm{MHz}$ in two vertical cut planes: (a) $\varphi=0^{\circ}$, (b) $\varphi=90^{\circ}$. RHCP in red, and LHCP in blue.

\section{CONCLUSION}

A compact RHCP metasurface-inspired cavity antenna $0.26 \lambda_{0} \times 0.26 \lambda_{0} \times 0.11 \lambda_{0}$, covering three different GNSS bands with a single radiating aperture, has been proposed at 1578 $\mathrm{MHz}$. The radiating aperture is loaded by a two-layer array of square patches excited by four feed ports in phase quadrature. This antenna concept has been introduced by first studying a similar antenna system, but operating in linear polarization. The LP antenna can be used onboard flying platforms in multipathfree-environments, while the $\mathrm{CP}$ version provides a better carrier-to-noise ratio $(C / N)$, which is also required for ground purposes.

The experimental results obtained in RHCP have shown an excellent agreement with the numerical predictions. The proposed antenna is able to sufficiently cover the three GNSS bands (L1, E1, G1) with an active gain of $16 \mathrm{dBic}$ and an axial ratio lower than $1.6 \mathrm{~dB}$ over this frequency range ([1550 1610] MHz).

\section{REFERENCES}

[1] J. Sanz Subirana, J. M. Juan Zornoza, and M. Hernández-Pajares, GNSS Data Processing, Vol. I: Fundamentals and Algorithms, Noordwijk: European Space Agency Communications, May 2013. 
[2] F. Mariottini, M. Albani, E. Toniolo, D. Amatori, and S. Maci, "Design a compact GPS and SDARS integrated antenna for automotive applications," IEEE Antennas and Wireless Propagation Letters, vol. 9, pp. 405-408, 2010.

[3] B. Rama Rao, W. Kunysz, R. Fante, and K. McDonald, GPS/GNSS Antennas, Artech House, 2013.

[4] Nasimuddin, Y. S. Anjani, and A. Alphones, "A wide-beam circularly polarized asymmetric-microstrip antenna", IEEE Trans. Antennas and Propag., vol. 63, no. 8, pp. 3764-3768, Aug. 2015.

[5] Nasimuddin, X. Qing, and Z. N. Chen, "A compact circularly polarized slotted patch antenna for GNSS applications", IEEE Trans. Antennas and Propag., vol. 62, no. 12, pp. 6506-6509, Dec. 2014.

[6] Y. Dong, H. Toyao, and T. Itoh, "Compact circularly-polarized patch antenna loaded with metamaterial structures", IEEE Trans. Antennas and Propag., vol. 59, no. 11, pp. 4329-4333, Nov. 2011.

[7] M. H. Cohen, "On the band width of cavity antennas," Journal of Applied Physics, vol. 25, no. 5, pp. 582-587, May 1954.

[8] F. Zavosh and J. T. Aberle, "Single and stacked circular microstrip patch antennas backed by a circular cavity," IEEE Trans. Antennas and Propag., vol. 43, no. 7, pp. 746-750, Jul. 1995.

[9] M. Lye, R. B. Waterhouse, D. Novak, F. Zavosh, and J. T. Aberle, "Design and development of printed antenna remote units for optically distributed mobile communications," IEEE Microw. and Guided Wave Letters, vol. 8, no. 12, pp. 432-434, Dec. 1998.

[10] L. Ge, and K. M. Luk, "A low-profile magneto-electric dipole antenna," IEEE Trans. Antennas and Propag., vol. 60, no. 4, pp. 1684-1689, April 2012.

[11] K. Agarwal, Nasimuddin, and A. Alphones, "Wideband circularly polarized AMC reflector backed aperture antenna," IEEE Trans. Antennas and Propag., vol. 61, no. 3, pp. 1456-1461, Mar. 2013.

[12] W. Yang and J. Zhou, "Wideband circularly polarized cavity-backed aperture antenna with a parasitic square patch," IEEE Antennas and Wireless Propag. Letters, vol. 13, pp. 197-200, 2014.

[13] N. C. Karmakar, "On miniaturisation and bandwidth enhancement of a cavity backed circular microstrip patch antenna," IEEE AP'S Int. Symp. Antennas and Propagation, San Antonio, TX, vol. 3, pp. 596-599, 2002.

[14] M. Martinis, K. Mahdjoubi, R. Sauleau, S. Collardey, and L. Bernard, "Circuit models explaining the bandwith behavior of small circular cavity-backed patch antennas," $8^{\text {th }}$ European Conference on Antennas and Propagation, EuCAP, The Hague, The Netherlands, 2014.

[15] M. Martinis, "Development and characterization of metamaterials in cavities. Applications to the design of compact antennas," $\mathrm{PhD}$ thesis, Université de Rennes 1, Rennes, 2014.

[16] L. García-Gámez, L. Bernard, R. Sauleau, S. Collardey, K. Mahdjoubi, P. Pouliguen, and P. Potier, "Metasurface-inspired antenna in cavity for GNSS applications," $12^{\text {th }}$ European Conference on Antennas and Propagation, EuCAP, London, UK, 2018.

[17] M. Martinis, K. Mahdjoubi, R. Sauleau, S. Collardey, and L. Bernard, "Bandwidth behavior and improvement of miniature cavity antennas with broadside radiation pattern using a metasurface," IEEE Trans. Antennas and Propag., vol. 63, no. 5, pp. 1899-1908, May 2015.

[18] M. E. de Cos and F. Las-Heras, "Polypropylene-based dual band CPW-fed monopole antenna," IEEE Antennas and Propag. Mag., vol. 55, no. 3, pp. 264-273, Jun. 2013.

[19] CST Studio Suite, www.cst.com.

[20] A. G. Koutinos, G. A. Ioannopouos, M. T. Chryssomallis, and G. A. Kyriacou, "Bandwidth enhancement of rectangular patch antennas using multiple feeding points: a review," $7^{\text {th }}$ International Conference on Modern Circuits and Systems Technologies, Thessaloniki, Greece, 2018.

[21] J.R. James and P. S. Hall, "Handbook of microstrip antennas," IEE electromagnetic waves series, 1989.

[22] M. Manteghi, and Y. Rahmat-Samii, "Multiport characteristics of a wideband cavity backed annular patch antenna for multipolarization operations", IEEE Trans. Antennas and Propag., vol. 53, no. 1, pp. 466474, Jan. 2005.

[23] Mini-circuits, "Power Splitter/Combiner," SCQ-4-1650+ datasheet.

[24] Infineon Technologies AG, "Silicon Germanium Low Noise Amplifier for Global Navigation Satellite Systems (GNSS) in ultra small package with $0.77 \mathrm{~mm}^{2}$ footprint," BGA725L6 datasheet, June 2011 [Revised Mars 2012].

[25] Mini-circuits, "Bias-Tee," TCBT-6G+ datasheet. 